\title{
Exploiting MET dysregulation in EGFR-addicted non-small-cell lung carcinoma: a further step toward personalized medicine
}

\author{
Vincenzo Di Noia ${ }^{1}$, Ettore D’Argento ${ }^{1}$, Sara Pilotto ${ }^{2}$, Miriam Grazia Ferrara ${ }^{1}$, Michele Milella ${ }^{2}$, \\ Giampaolo Tortora ${ }^{1}$, Emilio Bria ${ }^{1}$ \\ ${ }^{1}$ U.O.C. Medical Oncology, Fondazione Policlinico Universitario Agostino Gemelli, IRCCS, Università Cattolica del Sacro Cuore, Rome, Italy; \\ ${ }^{2}$ U.O.C. Oncology, Azienda Ospedaliera Universitaria Integrata, University and Hospital Trust of Verona, Verona, Italy \\ Correspondence to: Prof. Emilio Bria. U.O.C. Medical Oncology, Fondazione Policlinico Universitario Agostino Gemelli, IRCCS, Università Cattolica \\ del Sacro Cuore, Rome, Italy. Email: emilio.bria@unicatt.it. \\ Comment on: Wu YL, Zhang L, Kim DW, et al. Phase Ib/II Study of Capmatinib (INC280) Plus Gefitinib After Failure of Epidermal Growth Factor \\ Receptor (EGFR) Inhibitor Therapy in Patients With EGFR-Mutated, MET Factor-Dysregulated Non-Small-Cell Lung Cancer. J Clin Oncol \\ 2018. [Epub ahead of print].
}

Submitted Dec 07, 2018. Accepted for publication Dec 14, 2018.

doi: $10.21037 /$ tlcr.2018.12.08

View this article at: http://dx.doi.org/10.21037/tlcr.2018.12.08

Patients affected by non-small-cell lung cancer (NSCLC) harboring epidermal growth factor receptor (EGFR) activating mutations derive a dramatic and essential clinical benefit from $E G F R$ tyrosine kinase inhibitors (TKIs) therapy in terms of activity, efficacy and quality of life $(1,2)$. Nevertheless, besides the important therapeutic impact of these targeted agents, EGFR-addicted diseases typically develop resistance under the selective pressure of TKIs, usually within 1 year of treatment. In $50-60 \%$ of patients, such acquired resistance to first- and second-generation EGFR TKIs is related to the acquisition of EGFR T790M mutation, a second-site 'on-target' point mutation which affects the adenosine triphosphate (ATP) binding pocket of $E G F R$ tyrosine-kinase domain. In this regard, osimertinib, a third-generation $E G F R$ inhibitor with prominent activity against both T790M resistance mutation and the standard activating EGFR mutations, represents a very active treatment for patients with EGFR T790M-dependent NSCLC who experienced disease progression after firstline EGFR TKIs therapy, as demonstrated in AURA1-2-3 studies (3-5). While the EGFR T790M-driven disease still mirrors the typical oncogene-addicted NSCLC, with the majority of tumors dramatically responding to osimertinib and deriving a significant delay in tumor progression, T790M negative patients represent a heterogeneous population whereas tumors with extremely different clinical and biological findings co-exist and where platinum- based chemotherapy remains the only available worldwide approved treatment option. For these reasons, such clinical scenario represents a challenging issue for physicians in clinical practice.

MET gene amplification is the second most important mechanism (5-20\% of patients) of acquired resistance to EGFR TKIs and in a half of cases concurrently occurs with T790M EGFR mutation (6). MET-dependent resistance is triggered by the activation of common downstream pathways to $E G F R$ receptor, directly by the homodimer formation or indirectly by trans-activating other tyrosine kinase receptors. This way EGFR signaling becomes redundant and, as preclinical studies suggest, targeting both receptors by adding an anti-MET agent to EGFR TKIs is required to obtain an effective antitumor activity (7). A series of anti-MET-agents, including TKIs or monoclonal antibodies (MoAbs), have been developed and tested in NSCLC patients. The outcomes of EGFR mutated population included in anti-MET trials are reported in Table 1 (8-12).

Wu et al. recently published a phase $1 \mathrm{~b} / 2$ study (13), in which the combination of the $M E T$ inhibitor capmatinib and the EGFR inhibitor gefitinib appears safe and active in patients with T790M-negative acquired resistance to prior EGFR TKI therapy carrying MET-dysregulation (amplification or overexpression). Different doses of capmatinib plus gefitinib $(250 \mathrm{mg})$ were examined in 


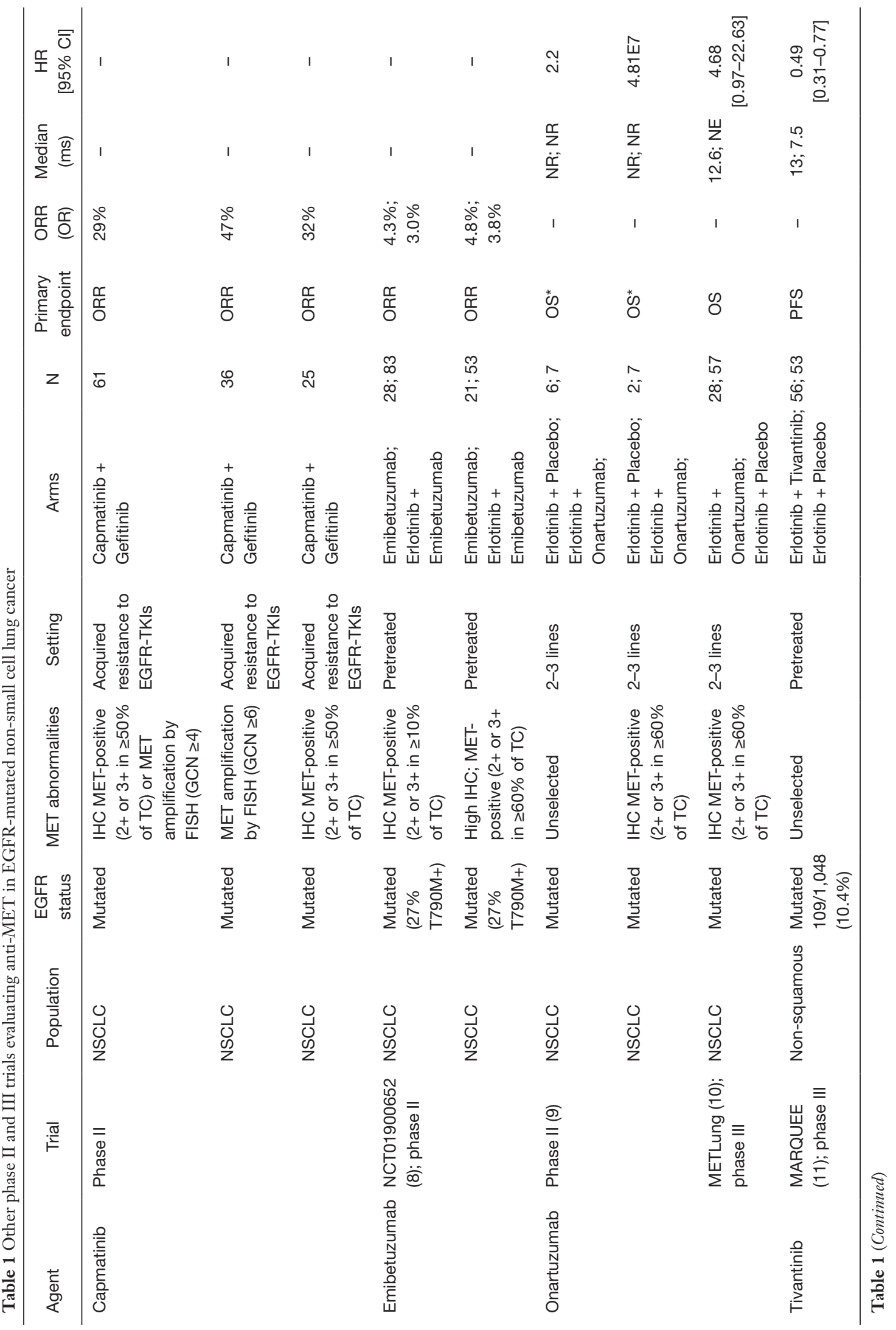




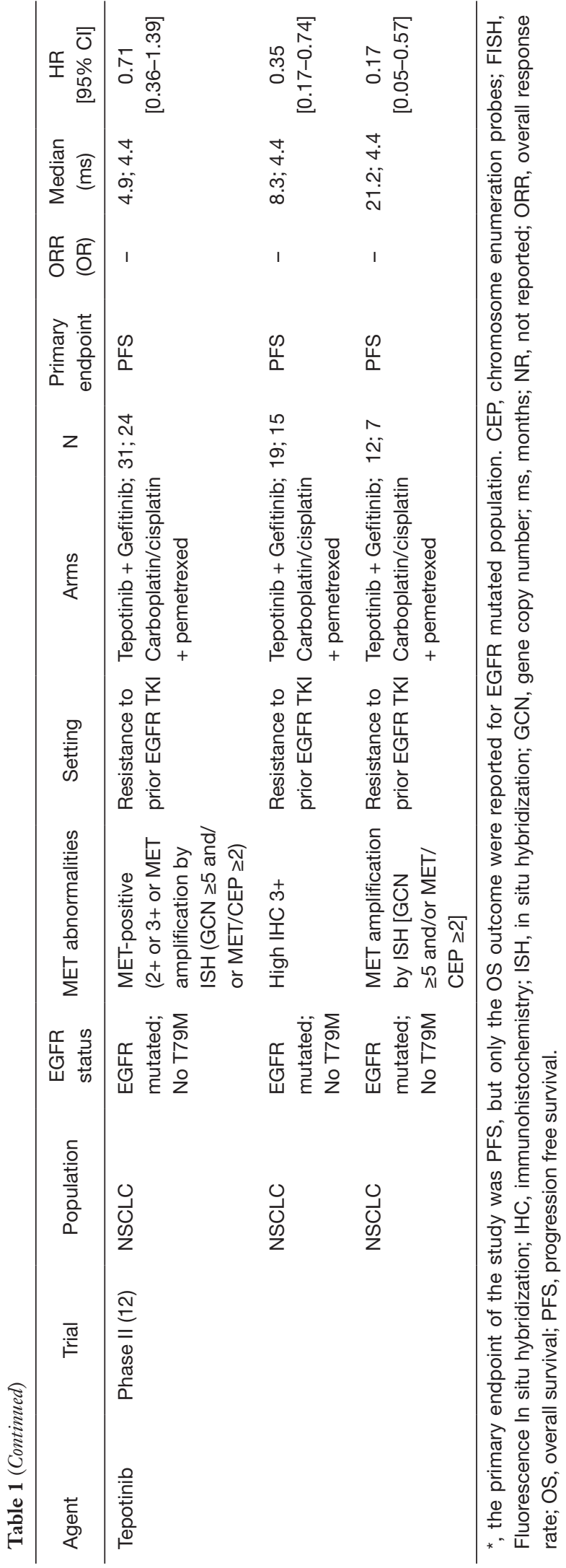

the phase $1 \mathrm{~b}$ part of the trial, in which 61 patients were enrolled. In the phase II of the trial, 100 patients received the combination of gefitinib (250 mg per day) plus capmatinib at the dose identified in the phase I part of the study (400 $\mathrm{mg}$ twice daily), and an overall response rate of $29 \%$ was achieved. Combining both phases, overall response rate was $27 \%$, with a generally well-tolerable safety profile of the combination. With regard to predictors of activity, a higher response rate $(47 \%)$ was observed in patients whose tumors harbor MET amplification with a gene copy number $(\mathrm{GCN}) \geq 6$; the ORR was $32 \%$ in patients with immunohistochemistry (IHC) score 3+ tumors, which was comparable with the observed activity in patients with IHC $2+$ and $\mathrm{GCN} \geq 5$ tumors. A trend towards a longer progression-free survival (PFS) was seen in patients with tumors with a GCN $\geq 6$ (5.49 months) or IHC2+/GCN $\geq 5$ (7.29 months). Thus, the biomarker analysis prioritizes MET fluorescence in situ hybridization (FISH) analysis (with a cut-off of GCN $\geq 6$ ) in comparison with MET IHC staining to identify tumors more likely to be shrank with the combination of capmatinib plus gefitinib.

These results, generate the hypothesis that a therapeutic strategy including the concurrent inhibition of MET and EGFR signaling could be conceivable for T790M negative NSCLC patients with MET induced acquired resistance to EGFR TKIs, who are currently candidate to receive platinum-based chemotherapy as standard treatment. In addition, these promising early data deserve to be preferentially investigated than those emerging from the addition of check-point inhibitors, whose efficacy appear to be limited in the oncogene-addicted disease, at least for now (14). With regard to chemotherapy, in the IMPRESS trial (15), non-squamous NSCLC patients who had progressed on first-line gefitinib were randomized to up to 6 cycles of cisplatin-pemetrexed chemotherapy together with continuation of the gefitinib or placebo. Patients who received chemotherapy alone achieved an ORR of $34 \%$ and a median PFS of 5.4 months, which were even lower in the plasma T790M negative population (ORR of $32 \%$ and PFS 4.6 months). Thus, although considering the bias of an indirect comparison, the activity of capmatinib and gefitinib seems to be at least comparable with chemotherapy in patients with acquired resistance to EGFR TKIs, and the hypothesis that this combination would better work in patients with $M E T$ amplification or overexpression is suggested.

Conversely to other studies including combination of targeted agents which were early stopped for unacceptable 
toxicity [ex. erlotinib plus tivantinib (16) and dacomitinib plus crizotinib (17)], the combination of capmatinib and gefitinib appears to be safe. The most common study drug-related any-grade and grade 3/4 adverse events were nausea (28\%) and increased lipase and amylase levels (both 6\%), respectively. Peripheral edema (all grades) was believed to be drug related and occurred in $22 \%$ of patients; that may be a potential drug-class effect since it has been peculiarly reported for other MET inhibitors as well.

Overall, the majority of trials investigating MET inhibition (either with TKIs or MoAbs) were prospectively conducted in non-EGFR-addicted disease, thus trying to interfere with a $M E T$ pathway dysregulation different from that emerging as an acquired mechanism of resistance in EGFR mutant NSCLC progressing during TKIs. Two phase III trials (MARQUEE and MET-Lung) were negative, although a signal of efficacy of tivantinib in combination with erlotinib (in comparison with erlotinib alone) is showed in the subgroup analysis performed in EGFR mutant patients of the MARQUEE trial (11). In this retrospective analysis, PFS was significantly longer in patients receiving the combination of TKIs, with a HR of 0.49 (95\% CI, 0.31-0.77), with a trend towards a longer OS as well (HR 0.68; 95\% CI, 0.43-1.08). In this subgroup analysis, $14 \%$ and less than $4 \%$ of patients showed MET overexpression or amplification, respectively, in the combination arm. All patients enrolled had received 1 or 2 prior line of chemotherapy regimens and no EGFRTKIs therapy. The most common adverse events of any grade were diarrhea, rash, and asthenia, which occurred at similar rates in the experimental arm $(39.3 \%, 30.4 \%$, $35.7 \%$, respectively) and in the control group (43.4\%, $30.4 \%, 39.6 \%$, respectively). A higher rate of both febrile neutropenia (3.6\%) and neutropenia (14.3\%) of grade $\geq 3$ was observed in patients receiving erlotinib and tivantinib.

In the trial of Wu et al., while patients were selected for acquired resistance to TKI according to the well-defined acquired resistance criteria (18), the definition of MET dysregulation, one of the key inclusion criteria, was amended overtime. Indeed, patients were sequentially required to have: (I) GCN $\geq 5$ or $50 \%$ of tumor cells with IHC $2+/ 3+$; (II) $50 \%$ of tumor cells with IHC $3+$ or IHC $2+$ plus MET GCN $\geq 5$; and finally $50 \%$ of tumor cells with IHC $3+$ or MET GCN $\geq 4$. Furthermore, in the early phase of enrollment $M E T$ dysregulation was assessed also in local laboratories, while finally, the central evaluation becomes mandatory.

Actually, the lack of a standard definition of c-Met dysregulation (as observed in this trial) suggests that several mechanisms, including amplification, rearrangements, protein overexpression and mutations are responsible for MET activation and probably determine different type and level of activation, which may induce different responses to specific therapeutic approaches. In addition to the biological issues, diagnostic tests and molecular biomarkers for patients' selection have never been established or validated. Wu et $a l$. had considered to evaluate $M E T$ abnormalities in terms of gene amplification by FISH and expression by IHC. Although the use of each technique is hampered by specific limitations, both assays deal with a continuous variable, and the selection of the cut-off points remains a crucial issue for the best selection of patients, since the ORR of capmatinib plus gefitinib increase directly and quantitatively with the rising of the cut-off considered. In particular, the evaluation of MET amplification by FISH was affected in first instance by the imperfect correlation between MET amplification and c-Met overexpression, which underline the potential influence of other mechanisms affecting gene expression (19). In addition, FISH cannot identify MET dysregulation arising from alterations other than MET amplification and it is technically limited by the small number of cells that can be feasibly assessed, resulting in a possible underestimation of true amplification of MET in heterogeneous tumors. This is particularly relevant for the assessment of tumors harboring small numbers of METamplified clones with the potential to expand under the selective pressure imposed by continued EGFR TKI therapy.

Even the IHC evaluation of $M E T$ expression required technical adjustments, such as careful sample handling, the inclusion of adequate controls, and the interpretation by experienced pathologists. Bypassing the technical issues, even in this case, $M E T$ levels cannot directly reflect the receptor activity, which is influenced also by the cross-interaction of MET with others TK receptors. Furthermore, tumors with similar levels of c-Met expression may differ in their sensitivity to c-Met inhibitors, depending on whether c-Met overexpression drives tumorigenesis, or is a bystander/passenger alteration.

A possible way of resolution of some (but not all) limitations related to MET IHC evaluation consists of the direct detection of phospho-c-Met- residues, which may be a more accurate indicator of $M E T$ activation than total MET-expression by IHC (20). Significant MET pathway inhibition was induced in 5 patients included in the trial, whose pre- and post-dose tumor samples were assessed for p-MET expression. Unfortunately, clinical outcome of the patients evaluated in this exploratory analysis were not 
reported by the authors and thus the two methods were not comparable for their predictive value.

Another interesting field of application of MET inhibition could be the landscape of acquired resistance to the thirdgeneration EGFR TKI osimertinib. Biomarker analysis performed on liquid biopsies in the FLAURA study demonstrated MET amplification as the most frequent mechanism of resistance to first-line osimertinib (15\% of evaluable patients) (21). A relevant role was attributed to MET amplification also in the acquired resistance to second-line osimertinib, as showed in a similar biomarker analysis of AURA3 study, in which MET amplification was the second cause of resistance (19\% of cases) after the loss of T790M EGFR mutation, detected in $49 \%$ of treated patients (22). Nevertheless, also the activity of the combination of MET inhibitors with third-generation EGFR TKIs seems to be promising. A response rate of $40 \%$ was recently reported for combinations of osimertinib and the MET-TKIS savolitinib in the phase Ib TATTON trial (23), including a cohort of patients with MET amplification assessed by FISH irrespective to T790M status. The targeting of both MET and EGFR pathway seems to be active also in treatment-naive patients unselected for $M E T$ status, considering the $47 \%$ of response observed with the combination of capmatinib plus the EGFR 3 th generation TKI nazartinib in a phase Ib/II study (24).

All these perspectives encourage further studies focused on anti-MET agents in different contexts of lung cancer. Probably, in the forthcoming future, considering the potential role of molecular MET testing as a predictor of efficacy of anti-MET therapy (25), it would be included in the routine panel of targetable molecular alteration of NSCLC. Before to move to such important step, the standardization of MET evaluation assays with the crucial definition of reliable (and reproducible) cutoffs is absolutely required to clearly identify those $M E T$ dysregulated NSCLC patients who would best benefit from $M E T$-targeted therapy.

\section{Acknowledgements}

Funding: Y Di Noia, E D'Argento, S Pilotto and E Bria are supported by the Italian Association for Cancer Research AIRC-IG 20583.

\section{Footnote}

Conflicts of Interest: The authors have no conflicts of interest to declare.

\section{References}

1. Lee CK, Davies L, Wu YL, et al. Gefitinib or Erlotinib vs Chemotherapy for EGFR Mutation-Positive Lung Cancer: Individual Patient Data Meta-Analysis of Overall Survival. J Natl Cancer Inst 2017;109.

2. Bria E, Milella M, Cuppone F, et al. Outcome of advanced NSCLC patients harboring sensitizing EGFR mutations randomized to EGFR tyrosine kinase inhibitors or chemotherapy as first-line treatment: a meta-analysis. Ann Oncol 2011;22:2277-85.

3. Jänne PA, Yang JC, Kim DW, et al. AZD9291 in EGFR inhibitor-resistant non-small-cell lung cancer. $\mathrm{N}$ Engl J Med 2015;372:1689-99.

4. Yang JC, Ahn MJ, Kim DW, et al. Osimertinib in Pretreated T790M-Positive Advanced Non-SmallCell Lung Cancer: AURA Study Phase II Extension Component. J Clin Oncol 2017;35:1288-96.

5. Mok TS, Wu YL, Ahn MJ, et al. Osimertinib or PlatinumPemetrexed in EGFR T790M-Positive Lung Cancer. N Engl J Med 2017;376:629-40.

6. Bean J, Brennan C, Shih JY, et al. MET amplification occurs with or without T790M mutations in EGFR mutant lung tumors with acquired resistance to gefitinib or erlotinib. Proc Natl Acad Sci U S A 2007;104:20932-7.

7. Wang W, Li Q, Takeuchi S, et al. Met kinase inhibitor E7050 reverses three different mechanisms of hepatocyte growth factor-induced tyrosine kinase inhibitor resistance in EGFR mutant lung cancer. Clin Cancer Res 2012;18:1663-71.

8. Wu YL, Zhang L, Kim DW, et al. Phase Ib/II Study of Capmatinib (INC280) Plus Gefitinib After Failure of Epidermal Growth Factor Receptor (EGFR) Inhibitor Therapy in Patients With EGFR-Mutated, MET FactorDysregulated Non-Small-Cell Lung Cancer. J Clin Oncol 2018. [Epub ahead of print].

9. Lee CK, Man J, Lord S, et al. Clinical and Molecular Characteristics Associated With Survival Among Patients Treated With Checkpoint Inhibitors for Advanced NonSmall Cell Lung Carcinoma: A Systematic Review and Meta-analysis. JAMA Oncol 2018;4:210-6.

10. Mok TS, Kim SW, Wu YL, et al. Gefitinib Plus Chemotherapy Versus Chemotherapy in Epidermal Growth Factor Receptor Mutation-Positive Non-SmallCell Lung Cancer Resistant to First-Line Gefitinib (IMPRESS): Overall Survival and Biomarker Analyses. J Clin Oncol 2017;35:4027-34.

11. Yoshioka H, Azuma K, Yamamoto N, et al. A randomized, 
double-blind, placebo-controlled, phase III trial of erlotinib with or without a c-Met inhibitor tivantinib (ARQ 197) in Asian patients with previously treated stage IIIB/IV nonsquamous nonsmall-cell lung cancer harboring wildtype epidermal growth factor receptor (ATTENTION study). Ann Oncol 2015;26:2066-72.

12. Jänne PA, Shaw AT, Camidge DR, et al. Combined PanHER and ALK/ROS1/MET Inhibition with Dacomitinib and Crizotinib in Advanced Non-Small Cell Lung Cancer: Results of a Phase I Study. J Thorac Oncol 2016;11:737-47.

13. Scagliotti GV, Shuster D, Orlov S, et al. Tivantinib in Combination with Erlotinib versus Erlotinib Alone for EGFR-Mutant NSCLC: An Exploratory Analysis of the Phase 3 MARQUEE Study. J Thorac Oncol 2018;13:849-54.

14. Jackman D, Pao W, Riely GJ, et al. Clinical definition of acquired resistance to epidermal growth factor receptor tyrosine kinase inhibitors in non-small-cell lung cancer. J Clin Oncol 2010;28:357-60.

15. Dziadziuszko R, Wynes MW, Singh S, et al. Correlation between MET gene copy number by silver in situ hybridization and protein expression by immunohistochemistry in non-small cell lung cancer. J Thorac Oncol 2012;7:340-7.

16. Arriola E, Cañadas I, Arumí-Uría M, et al. MET phosphorylation predicts poor outcome in small cell lung carcinoma and its inhibition blocks HGF-induced effects in MET mutant cell lines. Br J Cancer 2011;105:814-23.

17. Papadimitrakopoulou VA, Wu Y, Han J, et al. Analysis of resistance mechanisms to osimertinib in patients with EGFR T790M advanced NSCLC from the AURA3 study. Ann Oncol 2018;29:mdy424.064.

18. Ramalingam SS, Cheng Y, Zhou C, et al. LBA50

Cite this article as: Di Noia V, D'Argento E, Pilotto S, Ferrara MG, Milella M, Tortora G, Bria E. Exploiting MET dysregulation in EGFR-addicted non-small-cell lung carcinoma: a further step toward personalized medicine. Transl Lung Cancer Res 2018;7(Suppl 4):S312-S317. doi: 10.21037/ tlcr.2018.12.08
Mechanisms of acquired resistance to first-line osimertinib: Preliminary data from the phase III FLAURA study. Ann Oncol 2018;29:mdy424.063.

19. Ahn M, Han J, Sequist L, et al. TATTON Ph Ib expansion cohort: Osimertinib plus savolitinib for pts with EGFRmutant MET-amplified NSCLC after progression on prior EGFR-TKI. J Thorac Oncol 2017;12:S1768.

20. Tan DS, Lee DH, Soo R, et al. Phase Ib Results from a Study of Capmatinib (INC280) + EGF816 in Patients with EGFR-Mutant Non-Small Cell Lung Cancer (NSCLC). J Clin Oncol 2017;12:S1264-5.

21. Camidge DR, Moran T, Demedts I et al. A randomized, open-label, phase 2 study of emibetuzumab plus erlotinib $(\mathrm{LY}+\mathrm{E})$ and emibetuzumab monotherapy (LY) in patients with acquired resistance to erlotinib and MET diagnostic positive (MET Dx+) metastatic NSCLC. J Clin Oncol 2016;34:9070.

22. Spigel DR, Edelman MJ, O'Byrne K, et al. Onartuzumab plus erlotinib versus erlotinib in previously treated stage IIIb or IV NSCLC: Results from the pivotal phase III randomized, multicenter, placebo-controlled METLung (OAM4971g) global trial. J Clin Oncol 2014;32:8000.

23. Spigel DR, Ervin TJ, Ramlau RA, et al. Randomized phase II trial of Onartuzumab in combination with erlotinib in patients with advanced non-small-cell lung cancer. J Clin Oncol 2013;31:4105-14.

24. Wu Y, Zhou J, Lu S, et al. Phase II study of tepotinib + gefitinib $(\mathrm{TEP}+\mathrm{GEF})$ in MET-positive $(\mathrm{MET}+)$ / epidermal growth factor receptor (EGFR)-mutant (MT) non-small cell lung cancer (NSCLC). Ann Oncol 2018;29:viii493-547.

25. Pilotto S, Carbognin L, Karachaliou N, et al. Tracking MET de-addiction in lung cancer: A road towards the oncogenic target. Cancer Treat Rev 2017;60:1-11. 